\title{
La diferencia entre los otros: homofobia en el discurso subalterno
}

\section{Roxana Reyes Rivas ${ }^{1}$}

\section{Resumen}

Recepción: 26 de mayo de 2016 / Aceptación: 17 de octubre de 2016

Se parte del Análisis Crítico del Discurso (ACD) para explicar cómo se utiliza dicho discurso para establecer prácticas de poder al interior de grupos subalternos. En efecto, un grupo en desventaja, como respuesta a la discriminación que recibe, puede utilizar y legitimar discursos hegemónicos-igualmente discriminatorios- de los que se pueda considerar titular, como lo son los del machismo y la homofobia. En este trabajo nos ocuparemos del discurso homofóbico y su articulación en un grupo subalternizado en Costa Rica: los nicaragüenses. Primero, se explicará cómo se establece la matriz de poder que legitima el discurso homofóbico y, de forma posterior, como ejemplo, se analizará el video "Enrique Flores habla de los ticos", del comediante nicaragüense Reynaldo Ruíz, para mostrar cómo este usa el discurso homofóbico para responder a la xenofobia costarricense y, a su vez, establecer una jerarquía por orientación sexual y género, tanto dentro, como fuera de su propio grupo, repercutiendo en la nacionalidad.

\section{Palabras clave}

Subalterno; alteridad; homofobia; masculinidad hegemónica; discurso hegemónico

\section{Abstract}

The use of language to establish practices of power within subaltern groups is explained departing from Critical Discourse Analysis (CDA). Indeed, a disadvantaged group can use and legitimize discriminatory hegemonic discourses to which it feels entitled -such as the ones stemmed in machismo and homophobia- in response to the discrimination it endures. This paper is focused on the homophobic discourse and its articulation by a subalternized group in Costa Rica, namely, Nicaraguans. First, it is explained how the matrix of power that legitimizes the homophobic discourse is settled. The video "Enrique Flores habla de los ticos" by the Nicaraguan comedian Reynaldo Ruíz will be used as an example to show how he uses homophobic discourse in response to Costa Rican xenophobia. At the same time, he establishes a hierarchy through sexual orientation and gender, within and outside his group as well, resounding in nationality.

1 Costarricense. Máster en Filosofía de Indiana University (Bloomington, Indiana, Estados Unidos). Profesora de Filosofía y Lógica en la Escuela de Ingeniería en Computación y en la Escuela de Ciencias Sociales del Instituto Tecnológico de Costa Rica (ITCR). Doctoranda en el Programa de Estudios de la Sociedad y la Cultura de la Universidad de Costa Rica. Correo: roxana_reyes@yahoo.com 


\section{Key words}

Subaltern; alterity; homophobia; hegemonic masculinity; hegemonic discourse

\section{Resumo}

Faz parte da Análise Crítica do Discurso (ACD) para explicar como ela é usada para estabelecer práticas de poder dentro dos grupos subalternos. Com efeito, um grupo desfavorecido em resposta à discriminação recebidas, pode usar e legitimar discursos hegemônicos e igualmente discriminatória que pode ser considerado titular, assim como o machismo e homofobia. Neste artigo, vamos abordar o discurso homofóbico e sua articulação em um grupo subalternizado em Costa Rica: os nicaragüenses. O artigo explica como a matriz de poder que legitima o discurso homofóbico é estabelecida. Serão utilizados, como exemplo, o vídeo "Enrique Flores fala dos Ticos" de o comediante nicaragüense Reynaldo Ruíz para mostrar como ele usou o discurso homofóbico para responder à xenofobia costariquenha e, por sua vez, estabelecer uma hierarquia de orientação sexual e de gênero, tanto dentro como fora do seu próprio grupo, afetando nacionalidade.

\section{Palavras chave}

Subalterno; alteridade; a homofobia; a masculinidade hegemônica; discurso hegemônico

¿Cómo podemos superar la idea de que los oprimidos son de alguna manera necesariamente virtuosos?

Estudios culturales centroamericanos en el nuevo milenio (Zimmerman \& Baeza Ventura, 2009, p. xx).

\section{Introducción}

En el Análisis Crítico del Discurso (ACD) se parte de un compromiso por desentrañar el uso del lenguaje, en particular, lo que Fairclough (2001) llama el discurso ${ }^{2}$ en el ejercicio del poder de grupos hegemónicos sobre grupos menos favorecidos o subalternos (van Dijk, 1986, citado en Wodak, 2003, p. 17). No obstante, los discursos hegemónicos encuentran su efectividad en el reconocimiento y adopción de los grupos discriminados. Más aun, es posible encontrar grupos subalternos que pueden decodificar y entender muy bien el poder que se manifiesta en un tipo de discurso, pero esos mismos grupos subalternos no necesariamente comprenden, en todo su alcance, el poder y la opresión que otros discursos ejercen contra terceros grupos, también discriminados.

En efecto, la pertenencia a un grupo subalterno no excluye a sus integrantes de pertenecer a otros grupos a su vez discriminados. Así pues, la mujer obrera práctica social”] (traducción propia de la autora del artículo, 2001, p. 16). 
puede sufrir discriminación no solamente por clase social, sino también por razón de género. Inclusive, puede ser discriminada por miembros de su misma clase por razón de género, pues el discurso hegemónico del patriarcado puede estar totalmente naturalizado dentro del grupo al que pertenece. De esta forma, hay prácticas sociales y sus respectivos discursos que constituyen a algunos grupos como hegemónicos más allá de la clase o la etnia. Este es el caso del patriarcado.

Ahora bien, este mismo proceso de instauración de un discurso hegemónico a través de todos los diferentes grupos que constituyen una sociedad, puede convertirse en un discurso unificador. Esto ocurre con los discursos que giran alrededor de la nacionalidad, los cuales pueden ser construidos de forma dicotómica; esto es, por contraste respecto de una alteridad ${ }^{3}$. Por ejemplo, en la actualidad, la nacionalidad costarricense -en mucho- se afirma y se refuerza mediante un discurso en donde el otro está constituido por trabajadores y trabajadoras que emigran desde Nicaragua.

Sin embargo, el que los y las nicaragüenses emigrantes se conviertan en un grupo discriminado, no significa que estos no puedan articular, a su vez, un discurso que dé respuesta a dicha discriminación. Es posible que, desde la subalternidad, un discurso defensivo contra un grupo hegemónico no constituya un acto de discriminación -aunque tome la forma de escarnio o injuria-, ya que un grupo subalterno no tiene el poder para poner en una situación de desventaja a un grupo hegemónico. Empero, en la articulación de ese discurso, dicho grupo puede estar legitimando otros discursos hegemónicos -de un cariz igualmente discriminatorio- de los que este se considere partícipe, por lo menos, de forma parcial, como sucede con el machismo y la homofobia.

En lo que sigue, me interesa explorar cómo, con respecto a ciertas diferencias, aquellos que están considerados como los otros, se constituyen en partícipes de prácticas sociales hegemónicas y prácticas discursivas correspondientes a estas que, a su vez, son constitutivas de otras formas de la alteridad que se convierten -en su imaginario- en dignas de desprecio, injuria y escarnio; de ahí el uso del epígrafe citado al principio de esta introducción. En consecuencia, resulta importante ver las formas que toman los discursos discriminatorios en los grupos subalternos, a fin de no romantizar e idealizar a estos grupos.

Para lo anterior, me referiré a la homofobia y el discurso que se articula alrededor de esta en grupos discriminados. En la próxima sección me ocuparé de ver cómo la construcción de la masculinidad -en el ámbito latinoamericano- pasa por una gran exacerbación de la homofobia. De forma posterior, me referiré de manera breve a algunas formas de incorporar el poder en el discurso, sobre todo en formas veladas. Asimismo, se procederá a demostrar cómo ciertas formas de la diferencia pueden establecer jerarquías y formas de opresión aun

3 A lo largo de este artículo se utilizará este término como sinónimo de lo que se ha dado por llamar "otredad". 
al interior de los grupos subalternos mismos. Para finalizar, me referiré al video de Enrique Flores -personaje interpretado por el humorista nicaragüense Reynaldo Ruíz- contra los hombres costarricenses, en respuesta a la xenofobia sufrida por los y las nicaragüenses en Costa Rica.

\section{Género, masculinidad y homofobia}

No se nace mujer, se llega a serlo

El segundo sexo (Beauvoir, 1999, p. 13).

Como es ya conocido, la ubicación, las funciones y el prestigio de las personas dentro de una sociedad, en cuanto hombres y mujeres, no obedecen, de forma necesaria, a razones estrictamente biológicas. En efecto, gran cantidad de literatura se ha escrito sobre la construcción social alrededor de las funciones y la posición que las personas deben ocupar de acuerdo con su sexo (de Beauvoir, 1999; Haraway, 1991; Lamas, 1997; Conway, Bourque y Scott, 1997). A esta construcción social se le ha llamado "género" o "sexo social” (Delphy, 2003)4.

De esta forma, si bien Simone de Beauvoir (1999) reflexionó acerca de la construcción social de lo que se considera "mujer"5, la teoría de género ha evolucionado para considerar que lo mismo se puede hacer para comprender la masculinidad, ya que esta también es considerada una construcción social. Así pues, en diferentes sociedades, encontramos distintas asignaciones para hombres y mujeres; en otras palabras, lo que se considera masculino o femenino puede variar de una colectividad a otra.

Ahora bien, en las sociedades latinoamericanas -incluidas las centroamericanas- como en muchas otras, la masculinidad hegemónica se asocia con el poder en tanto dominio. Además, lo masculino debe ser entendido como totalmente opuesto a todo lo que se considere como femenino. Es decir, si dentro del género femenino se han ubicado características como la domesticidad, la afectividad y el ser nutricia, con el género masculino se asocian las características de la publicidad, el desapego y la competitividad.

4 Aquí solamente me referiré a una versión muy general de la teoría del sistema sexo/género, donde normalmente se asume que se construyen dos géneros: femenino y masculino. No obstante, desarrollos posteriores han cuestionado un sistema tan dicotómico y han propuesto que existen más de dos géneros específicos (Haraway, 1991; Buttler, 1996). No discutiré esto, pues está fuera del alcance de este trabajo.

5 De hecho, de Beauvoir considera que esas construcciones sociales solamente rigen con respecto de las mujeres, ya que estas son constituidas como tales de acuerdo con discursos y prácticas que las perciben como la alteridad en un mundo donde los hombres son el modelo y medida de las cosas; no hay reciprocidad (de Beauvoir, 1999, p. 49). Sin embargo, en estudios más recientes, se ha mostrado cómo tampoco la masculinidad es homogénea y obedece, a través de diferentes sociedades, a lógicas de construcción similares a las de la feminidad, aunque normalmente con resultados, en términos valorativos, más favorables. 
Además, en lo que se refiere tanto a la feminidad como a la masculinidad hegemónicas, estas se encuentran determinadas por el mandato de la heterosexualidad' ${ }^{6}$. De esta forma, la peor falta a la hombría o masculinidad es ser gay u homosexual; de ahí que el insulto más grave que se puede proferir contra un varón -al menos desde una óptica hegemónica- es la de llamarle homosexual por cualquiera de las formas peyorativas que el habla popular ha acuñado. En efecto, gran cantidad de discursos, desde la ciencia hasta la comedia, se articulan alrededor de la homosexualidad masculina, ya sea en un intento de explicarla o, como en el caso de la comedia y el chiste, en un ejercicio de descalificación que pueda actuar como advertencia en contra de la práctica.

No obstante, si bien la homosexualidad masculina es una trasgresión profunda a las determinaciones de género, no todos los grupos, en las diferentes sociedades, tienen una misma percepción sobre lo que se puede definir por homosexualidad masculina. Así, en algunas sociedades o grupos dentro de estas, se le define por el objeto sexual, mientras que en otros ámbitos se le determina por la finalidad. Lo anterior implica que, en algunos casos, la homosexualidad está determinada por el sexo de la persona con que se realice el acto sexual; en otra, por el tipo de acto que se lleve a cabo. En el ámbito latinoamericano, de acuerdo con Mariana Castañeda,

el acto sexual característico de la masculinidad es penetrar, sea cual sea el sexo de la persona ... todo hombre que se deja penetrar automáticamente se asimila (y 'se rebaja') a la posición de la mujer. Como, además, en este sistema el homosexual se define como un hombre femenino, lógicamente el hombre penetrado es femenino y, por lo tanto, homosexual, mientras que quien penetra continúa siendo hombre y, según él, heterosexual -aunque tenga relaciones sexuales con otros hombres (Castañeda, 1999, p. 41).

En sociedades profundamente machistas como las latinoamericanas, lo que se percibe y define como femenino, en muchos casos, se desvaloriza ${ }^{7}$. En este contexto, se entiende que un hombre que tome una posición o actitud supuestamente femenina "se rebaje" al hacerlo. De esta forma, también se hace explícito cómo la homofobia y la misoginia son connaturales a la heterosexualidad masculina hegemónica ${ }^{8}$.

\footnotetext{
6 Aunque en lo que se refiere a la heterosexualidad de la mujer, esta posiblemente tenga su mayor importancia en cuanto se percibe, por un lado, como la condición para la maternidad, atributo cumbre de la feminidad hegemónica; por otro, a la disponibilidad del cuerpo femenino para el placer del hombre heterosexual.

7 Excepto, posiblemente, la maternidad.

8 Carlos Sandoval (2006, pp. 148-153) ha mostrado cómo la mención de la penetración de un hombre por otro hombre se usa como una metáfora del honor disminuido.
} 


\title{
Discurso y poder
}

\author{
[a] través de los chistes racializados \\ se clasifica, califica y coloca en una posición \\ de desventaja, exclusión e inferioridad \\ a las personas ... esto es control social, \\ pues recordemos que al clasificar y contener, \\ tenemos la sensación de que podemos controlar \\ "Chistes sobre nicaragüenses en Costa Rica" \\ (Masís y Paniagua, 2007, p. 342).
}

En el análisis crítico del discurso (ACD) no solamente se toma en cuenta el contexto de los discursos, sean estos hablados o escritos, sino los valores, la ideología de quienes los emiten, ya sean instituciones, grupos o individuos. Además, este se ocupa de entender cómo ciertos discursos sirven para poner o mantener en desventaja a individuos o grupos humanos. En palabras de van Dijk:

En vez de centrarse en problemas puramente académicos o teóricos, su punto de partida se encuentra en los problemas sociales predominantes, y por ello escoge la perspectiva de quienes más sufren para analizar de forma crítica a quienes poseen el poder, a los responsables, y a los que tienen los medios y la oportunidad de resolver dichos problemas (van Dijk, 1986, citado por Wodak, 2003, p. 17).

En otras palabras, quienes se dedican al ACD entienden que el lenguaje no puede ser considerado como un fenómeno aislado, sino que este ocurre en un ámbito o contexto sociocultural. Más aun, ambos -el contexto sociocultural y el lenguaje- se afectan de forma mutua, mas no de forma simétrica, ya que los fenómenos sociales no son estrictamente lingüísticos, aunque tienen una dimensión lingüística (Fairclough, 2001, pp. 18-20).

Ahora bien, una vez mencionado lo anterior, se puede afirmar que las relaciones de poder -el ejercicio del poder en tanto lugar de dominio- posee prácticas discursivas correspondientes. De hecho, es difícil concebir el ejercicio del poder sin un conjunto de prácticas que lo refuercen y lo reproduzcan. Así, desde las instituciones del Estado, las instancias del gobierno y otras entidades, hasta las relaciones interpersonales, podemos ver cómo se articulan discursos mediante los cuales se ejercen diferentes tipos de dominio, al tiempo que son reforzados y perpetuados. No obstante, eso no significa que no pueda haber cambios en las relaciones de poder y en las prácticas discursivas. En palabras de Fairclough:

I have been suggesting that orders of discourse embody ideological assumptions, and these sustain and legitimize existing relations of power. If there is a shift in power relations through social struggle, one can expect transformation of orders of discourse. Conversely, if power relations remain relatively stable, this may give a conservative quality to reproduction?

9 He estado sugiriendo que los órdenes del discurso encarnan supuestos ideológicos y estos sostienen y legitiman relaciones de poder existentes. Si hay un cambio en las relaciones de poder a través de la lucha social, uno puede esperar una transformación en los órdenes del discurso. Inversamente, 
En efecto, los discursos que perpetúan relaciones de dominio y, por ende, de desigualdad -ya sean velados o explícitos- son diversos y se intercalan de manera compleja. En este sentido, en una misma pieza discursiva es posible encontrar no solamente formas de justificación de un poder político ilegítimo, sino también de racismo. Es el caso del canciller del gobierno golpista de Honduras, Enrique Ortez Colindres, cuando, en referencia al presidente estadounidense Obama, declaró: "En primer lugar el presidente de la República ... que lo respeto al negrito, no sabe dónde queda Tegucigalpa" (29 de junio de 2009, 0:14"-0:21").

En otras palabras, los discursos del racismo, el sexismo, la homofobia, el machismo, entre otros, son discursos de poder, primero, porque intentan establecer desigualdades entre las personas; segundo, porque ese mismo discurso, muchas veces, intenta legitimar las desigualdades; tercero, porque, a la luz de esas desigualdades se justifica el hecho de que algunos sean considerados mejores que otros; finalmente, a raíz de lo anterior, se justifica y legitima que algunos ejerzan el dominio sobre otros.

Resulta posible que una de las prácticas discursivas del poder más contundentes sea el chiste. Como lo menciona el epígrafe de esta sección, cierto tipo de chistes -en este caso, el racializado- puede fortalecer prácticas de poder de un grupo sobre otro, pues reduce a unos mientras que, por contraste, hace aparecer a otros como superiores. En el caso particular de Costa Rica, eso se concreta mediante los "chistes de nicas" (Ramírez, 2007; Masís y Paniagua, 2007).

El problema radica en que, con respecto al humor, se tiende a ejercer menos crítica. En efecto, es normal considerar este hecho inofensivo, ya que "no es en serio"10. Por tanto, a través del chiste, se manifiesta una licencia para externar pensamientos que no se dirían en un contexto real y serio. En consecuencia, en muchos casos, mediante esta práctica se refuerzan y expresan ideas, estereotipos y prejuicios. 


\title{
Diferencia y jerarquía entre los otros
}

\author{
Homophobia is usually the last oppression to be \\ mentioned, the last to be taken seriously, the last to go. \\ But it is extremely serious, sometimes to the point of being fatal..." \\ "Homophobia: Why Bring It Up?" (Smith, 1993, p. 99).
}

Ahora bien, como se mencionó, las relaciones de poder son complejas. Estas no se ejercen únicamente entre personas de diferentes grupos, sino que ocurren dentro de los miembros de una misma colectividad; aun al interior de los grupos que consideramos los otros puede haber relaciones de poder que propicien prácticas discriminatorias con sus correspondientes discursos. Por tanto, existen múltiples diferencias por medio de las cuales es posible establecer jerarquías, mediante el recurso de devaluar a quienes consideramos los otros o los diferentes. Así, la discriminación de algunos grupos a causa de lo que se considera diferente ha sido más naturalizada que otras y, en ese orden, es factible mencionar el machismo y la homofobia. En efecto, las prácticas y discursos que naturalizan la supuesta inferioridad de las mujeres frente a los hombres han sido de las más persistentes en diferentes sociedades y culturas.

Lo mismo ocurre con la homofobia contra hombres y mujeres ${ }^{12}$. Ciertamente, el odio y el miedo irracionales contra quienes no cumplen con el mandato de la sexualidad normalizada, son considerados, en muchos casos, como reacciones naturales; es abominación en la mayoría de los discursos que se valoran como serios y con ascendiente moral o científico sobre los miembros de un grupo y, como consecuencia, la homofobia termina por convertirse en tabú dentro de algunos movimientos políticos que reclaman derechos para otros grupos discriminados o minorizados.

En concordancia con lo anterior, el discurso de grupos políticos organizados no se concreta en una práctica que la neutralice. Así, el epígrafe de esta sección proviene de un artículo de Barbara Smith (1993), donde la autora reclama la falta de consistencia de las feministas negras frente a la homofobia, ya que estas no actuaban acorde a su discurso cuando se presentaban brotes de homofobia en sitios públicos. En otras palabras, aun en una posición de precariedad, se puede señalar a alguien más como el otro. No obstante, a pesar de ser un punto de suma relevancia, se encuentra fuera del alcance de este trabajo comprender la persistencia de ese tipo de discriminaciones a través de otras variables como clase, etnia y edad.

11 La homofobia es usualmente la última opresión en ser mencionada, la última en ser tomada en serio, la última en terminar. Pero es extremadamente seria, a veces al punto de ser fatal (traducción propia de la autora del artículo, Smith, 1993, p. 99).

12 En algunos discursos políticos por los derechos de grupos minorizados por orientación sexual se usa "homolesbofobia" como una manera de designar dicha práctica. Por lo demás, en mucha literatura se prefiere la denominación "homoerotismo" u "homofilia", en vez de "homosexualidad", pues se considera que esta última alude a una perspectiva muy genital y sexualizada de la misma (Kennedy, 1997, p. 42, n. 14), aparte de tener connotaciones clínicas. Otros discursos políticos como el del movimiento queer aboga por eliminar categorías específicas (Castañeda, 1999, p. 25; Warner, 1994, pp. xii-xiv). 


\title{
Un ejemplo
}

\begin{abstract}
Por lo demás, ya las recetas callejeras de oprobio ofrecen una ilustrativa maquette ... El hombre de Corrientes y Esmeralda adivina la misma profesión en las madres de todos, o quiere que se muden en seguida a una localidad muy general que tiene varios nombres, o remeda un tosco sonido "Arte de injuriar" (Borges, 1998, p. 168).
\end{abstract}

Nicaraguan News Network es un programa televisivo de humor político que se transmite en el Canal 2 de Nicaragua. Este tiene el formato de un noticiero; inclusive, su logo tiene una gran similitud con el de la cadena de noticias estadounidense CNN. No obstante, como se indicó, las supuestas noticias que se presentan son, en realidad, sketches humorísticos. Uno de los comediantes que presenta esto segmentos es Reynaldo Ruíz. Para efectos de este trabajo, interesa mencionar un video actuado por dicho actor. El género discursivo que corresponde aquí es el de la comedia. Al ser escenas de corta duración, estas son representaciones teatrales de farsa. En efecto, el espacio en el que se supone se desarrollan los hechos mostrados es una barbería. En ella, Reynaldo Ruíz aparece como un barbero -Enrique Flores-, que lanza diatribas con respecto a varios asuntos.

Es interesante que el espacio sea una barbería, ya que se considera un lugar de sociabilidad masculino por excelencia. Mientras que las mujeres arreglan su cabello en un salón de belleza, los varones lo hacen en una barbería. Así pues, en este espacio de homosocialidad masculina -en el que se permite entrar por medio del video- se puede observar el intercambio entre hombres. A su vez, cuando el rostro del actor se dirige directamente hacia la cámara -como hablando para el público-, el tipo de discurso verbal que articula y el lenguaje corporal, siguen dando la impresión de que se dirige a otros hombres. Más abajo me ocuparé de esto.

El video que me interesa discutir se encuentra en YouTube ${ }^{13}$ y se intitula "Enrique Flores habla de los ticos". Después de una breve presentación desde el supuesto set de NNN, se da el pase a la barbeńa de Enrique Flores. Hay una pequeña introducción donde el personaje entra con tijeras y peine de barbero a cortarle el pelo a otro personaje que aparece sentado en la silla de barbería y conversa un par de frases con el presunto cliente. Posteriormente explica:

Hombre ando arrecho porque me caen mal estos ticos maricones. Me caen mal estos ticos maricones. Porque los ticos son maricones iverdad? Se la lanzan de gran cosa y ni siquiera saben trabajar. Porque quienes llegamos allá, a Costa Rica a sembrarles el plátano somos nosotros los nicaragüenses (NNN, s.f., 0:15"-0:29").

Este fragmento de discurso expresa, de forma evidente, una gran homofobia. La palabra "maricón" no solamente denota a un hombre homosexual, sino a uno que es imaginado como afeminado. Lo anterior se ve reforzado con

13 Se puede encontrar en la siguiente dirección electrónica http://www.youtube.com/watch?v=aNuOWaa7ftg 
la última afirmación; en efecto, la expresión "sembrar el plátano" juega con un recurso muy común dentro del lenguaje humorístico, a saber, el doble sentido sexual. En primer lugar, menciona el trabajo agrícola que muchos nicaragüenses realizan en Costa Rica; sin embargo, dicha expresión también alude a la penetración efectuada en un hombre por otro hombre. Como se mencionó, la lógica de este discurso sostiene que el hombre nicaragüense penetra al hombre costarricense quien, precisamente, por permitir dicha penetración, es un maricón y, por tanto, es débil e inferior, pues se coloca en una posición supuestamente femenina. Por otra parte, el nicaragüense afirma su hombría en el acto de penetrar y "rebajar" al hombre tico.

En otra parte del mismo sketch, el personaje menciona: “... porque con tanto nica que hay allá en Costa Rica, cuando se arme la guerra [ruido y gesto que normalmente indica relaciones sexuales $]^{14}$ los agarramos y les ganamos a estos ticos" (NNN, s.f., 0:34"-0:38"). Aquí, de igual manera, la alusión es claramente sexual. En este segmento palabras como "ganar", "vencer" y "humillar" están asociadas a las relaciones sexuales que, de acuerdo con el gesto, sugiere nuevamente la penetración por parte de los hombres nicas a los hombres ticos.

Más adelante, después de quejarse de la xenofobia contra los nicaragüenses en Costa Rica, dice:

¿Por qué a los colombianos no les hacen ese mate? ¿Ah? ¿Por qué a los colombianos no les salen con esas babosadas? A los colombianos los andan chinchineando y hasta les andan sobando las, las, las zapatillas, ijodido! ¿me entendés? Claro, me imagino que Colombia [gesto que insinúa relaciones sexuales] algún polvito le da a Costa Rica. Por eso es que no les hacen ningún mate a los colombianos, idejate de babosadas! (NNN, s.f., 1':22"'-1':38").

Aquí, el "polvito" puede, del mismo modo, interpretarse de dos maneras: una, viniendo de Colombia -y haciendo una asociación estereotipada de ese país con el narcotráfico- puede vincularse con la cocaína; dos, "polvo" también significa tener relaciones sexuales. De esta forma, la insinuación consiste en que los colombianos, de igual forma, tienen relaciones sexuales con los ticos, donde estos últimos son los maricones.

En otras partes de su sketch repite el calificativo de maricones para los ticos, la manera en que los nicas

aparte de que les llegamos a sembrar el plátano y el banano y se los sembramos bien sembraditos porque no sirven, ni se les para [pitido] ${ }^{15}$, ni hacen nada, somos nosotros los que llegamos a Costa Rica y agarramos a las ticas, las mujeres de ellos, porque idejate de babosadas! los ticos son playillos y ellas son unas grandes [¿putas? pitido]. Entonces ¿quiénes llegan a Costa Rica y agarran a las mujeres de los ticos? ¡nosotros! jnosotros! Les pegamos una gran [pitido] jvieras cómo

14 Uso aquí corchetes para indicar algo que supongo es parte del discurso, aunque no sea verbal, en este, caso los gestos o lenguaje corporal.

15 Normalmente usado en los medios de comunicación cuando se profiere una obscenidad. 
agarramos a esas ticas y hasta que gritan! [gestos con las manos que insinúan relaciones sexuales] (NNN, s.f., 2':27'-2':52').

El pasaje recién citado, aparte de continuar con su prédica homofóbica, tiene una gran carga machista y hace patente que el pleito contra los ticos es, en todo caso, entre hombres, aunque algunos de ellos sean considerados maricones y, por consiguiente, inferiores. Así pues, la supuesta ofensa se completa mediante el acceso carnal a las mujeres que, se supone, son propiedad de los ticos, aunque estos sean maricones. Además, en la totalidad del sketch, el discurso contra los ticos ostenta un gran machismo, ya que se asume que quien permite la penetración se pone en una situación abyecta, pues se feminiza. En otras palabras, lo que se percibe como femenino es abyecto.

A partir del análisis anterior, es posible argumentar que mediante el humor y el chiste se intenta descalificar, por parte de los nicaragüenses, a quienes se han constituido en sus opresores. Ya que el sketch es trasmitido en Nicaragua y difundido por Internet, quienes lo interpretan, no se encuentran en la situación de desventaja que la que se ubican los nicaragüenses en Costa Rica. Es muy posible que la intención del comediante sea la de convertirse en una voz de protesta y enojo para los nicaragüenses que viven en condiciones de desventaja y humillación en el país costarricense. A fin de lograrlo, echa mano de lo que Borges (1998) llamó "las recetas callejeras de oprobio".

No obstante, el efecto de descalificación y ofensa es poco en los y las nacionales costarricenses, debido a que los y las nicaragüenses, dentro de Costa Rica, no tienen el poder simbólico para causar mella a la autoimagen de supuesta superioridad de los y las costarricenses frente a los y las nicaragüenses ${ }^{16}$. Por el contrario, lo interesante consiste en detectar cómo la homofobia y el machismo se convierten en formas legítimas de humillar a otros por encima de la clase, nacionalidad o etnia. En otras palabras, aun los grupos más oprimidos pueden encontrar una forma de discriminar a los otros.

Así pues, aún aquellos que son considerados los otros, encuentran diferencias que les permiten a construir otras alteridades, inclusive, teniendo en común discursos con grupos hegemónicos que los oprimen. En efecto, la homofobia, el machismo y el sexismo son formas de construir alteridades que pueden ser compartidas entre grupos hegemónicos y subalternos. En este sentido -al respecto de ciertas construcciones de la alteridad- lo que puede cambiar a través de la clase, la nacionalidad o la escolaridad, es la forma que toma la expresión del oprobio

16 Poco antes de finalizar la corrección de este artículo, en marzo de 2016, el Ministerio de Seguridad Pública, a través de la Dirección General de Migración y Extranjería de Costa Rica, prohibió el ingreso del mencionado comediante, alegando no poder asegurar su integridad física, debido a la indignación que sus sketches han producido en algunos costarricenses. De acuerdo con la publicación del 10 de marzo de 2016 del periódico La Nación: "Esta orden, según el MSP, se basa en el artículo 61 de la Ley General de Migración y Extranjería, el cual dice que en el tanto "existan motivos fundados para considerar que su ingreso compromete la seguridad pública se puede impedir el ingreso al país...". Cabe preguntarnos si estas medidas pueden leerse también como una reafirmación de la construcción de la autoimagen costarricense arriba mencionada. 
o la injuria. Por ejemplo, mientras Borges, en su recensión de "La balada de la cárcel de Reading" (1998, pp. 130-133), hace gala de su homofobia eludiendo mencionar la homosexualidad cuando escribe sobre los infortunios de Oscar Wilde y alude burlonamente a Cocteau en el mismo escrito ${ }^{17}$, Reynaldo Ruíz, a través de su personaje Enrique Flores, se vale de palabras y gestos soeces para expresar la suya.

\section{Conclusiones}

En este trabajo se ha mostrado cuál es el contexto en que el comediante nicaragüense Reynaldo Ruíz, a través de su personaje Enrique Flores, articula su discurso homofóbico y machista contra los hombres costarricenses y -de manera eventual- también contra las mujeres costarricenses. Este contexto va más allá de una simple situación de tensión entre los habitantes de dos países vecinos. Ciertamente el detonante consiste en la situación discriminatoria en que viven los y las emigrantes nicaragüenses en Costa Rica y, de forma principal, los discursos que reproducen y refuerzan esta coyuntura, de modo que se entiende la intención explícita del mencionado humorista de contrarrestar y responder a esa situación. No obstante, he mostrado que, a su vez, el discurso que se articula en la pieza de comedia examinada logra, asimismo, reproducir otros discursos discriminatorios frente a otras diferencias. Así, finalmente, el hombre heterosexual y sexualmente agresivo se erigirá siempre en un tipo superior de ser humano por sobre todos lo demás. La diferencia de la homosexualidad y de la feminidad justifica, entonces, la discriminación y la abyección entre los otros.

Es importante desentrañar cuáles son las diferentes formas en que los seres humanos podemos lastimar a nuestros semejantes. El discurso es una de las maneras más poderosas dentro de la práctica social. Examinar esos discursos cuando son articulados al interior de grupos en desventaja es una buena práctica, ya que conduce a la autocrítica y a neutralizar otras formas de discriminación. En efecto, el que un individuo o grupo entienda su propia condición de discriminación no lo exime de ejercer otras; así, por ejemplo, es posible, de igual manera, encontrar personas de la población LGTB racistas, clasistas o con prácticas xenofóbicas. En otras palabras, aun dentro de los grupos en desventaja, se establecen jerarquías ora por género, ora por orientación sexual, ora por etnia, ora por nacionalidad.

Dentro de los diferentes discursos, uno de los más usados y más difíciles de neutralizar es el que se funda en el humor. Mediante el chiste, la chota y la comedia, muy fácilmente colocamos al otro en una situación de desventaja.

17 Afirma Borges sobre Wilde: "Su actividad fue comparable a la que hoy ejerce Cocteau, si bien su gesto luce más suelto y travieso que el del citado francesito" (Borges, 1998, p. 131) (el énfasis es de la autora del artículo). 
Es, pues, importante tener en cuenta de qué nos reímos y qué forma se toma esa risa, para entender si, mediante esta, estamos contribuyendo a maltratar a otros y, a veces, hasta a aniquilarles.

\section{Referencias}

Álvarez Casildo, Celeo. (11 de julio de 2009). Denuncia en contra del Señor Enrique Ortez Colindres. Recuperado de http://todo.honduraslaboral.org/ leer.php/1952.html

Beauvoir, Simone de. (1999 [1949]). El segundo sexo (Vols. I y II, traducción de Alicia Martorel). Madrid: Ediciones Cátedra.

Borges, Jorge Luis. (1989 [1926]). La balada de la cárcel de Reading. En El tamaño de mi esperanza (pp. 130-133). Madrid: Alianza Editorial S.A.

Borges, Jorge Luis. (1998 [1936]). Arte de injuriar. En Historia de la eternidad (pp. 167-177). Madrid: Alianza Editorial S.A.

Bringmann, Klaus. (1994 [1984]). El triunfo del emperador y las Saturnales de los esclavos en Roma. En Uwe Schultz (dir.) La fiesta. De las Saturnales a Woodstock. Traducción de José Luis Gil Aristu (pp. 7-22) Madrid: Alianza Editorial S.A.

Buttler, Judith. (1997 [1982]). Variaciones sobre sexo y género: Beauvoir, Wittig y Foucault. Traducción de Ana Sánchez. En Marta Lamas (comp.), El género: la construcción cultural de la diferencia sexual (pp. 303-326) México: Programa Universitario de Estudios de Género.

Castañeda, Marina. (1999). La experiencia homosexual. Para comprender la homosexualidad desde dentro y desde fuera. México: Editorial Paidós.

Conway, Jill K.; Bourque, Susan C. y Scott, Joan W. (1997 [1987]). El concepto de género. Traducción de Claudia Lucotti. En Marta Lamas (comp.), El género: la construcción cultural de la diferencia sexual (pp. 21-33). México: Programa Universitario de Estudios de Género.

Dijk, Teun A. van. (2003). El discurso como interacción en la sociedad. Traducción de José Ángel Álvarez. En Teun A. van Dijk (comp.) El discurso como interacción social. Estudios sobre el discurso II. Una introducción multidisciplinaria (pp. 19-66). Barcelona: Editorial Gedisa S.A.

Fairclough, Norman. (2001). Language and Power (segunda edición). Inglaterra: Longman.

Haraway, Donna J. (1991). Simians, Cyborgs, and Women. The Reinvention of Nature (cap. 7). New York: Routledge. 
Kennedy, Hubert. (1997). Karl Heinrich Ulrichs. First Theorist of Homosexuality. En Vernon Rosario (ed.) Science and Homosexualities (pp. 26-45). New York: Routledge.

Lamas, Marta. (1997 [1986]). Usos, dificultades y posibilidades de la categoría 'género'. En Marta Lamas (comp.) El género: la construcción cultural de la diferencia sexual (pp. 327-364). México: Programa Universitario de Estudios de Género.

Masís Fernández, Karen y Paniagua Arguedas, Laura. (2007). Chistes sobre nicaragüenses en Costa Rica: barreras simbólicas, mecanismos de control social, constructores de identidades. En Carlos Sandoval G. (ed.) El mito roto. Inmigración y emigración en Costa Rica (pp. 339-355). San José: Editorial Universidad de Costa Rica.

Mendez, Alejandro (10 de marzo de 2016). Migración prohíbe ingreso de humorista nicaragüense Reynaldo Ruíz que se burla de los ticos. La Nación. Recuperado de http://www.nacion.com/nacional/Migracion-humorista-nicaraguense-Reynaldo-Ruiz_0_1547645298.html

Ortez Colindres, Enrique. (29 de junio de 2009). Entrevista de Renato Álvarez [video]. Entrevista con Enrique Ortez Colindres. Recuperado de https:// www.youtube.com/watch?v=BVVixfNyyK8

Ramírez Caro, Jorge. (2007) El chiste de la alteridad: la pesadilla de ser otro. En Carlos Sandoval G. (ed.) El mito roto. Inmigración y emigración en Costa Rica (pp. 313-337). San José: Editorial Universidad de Costa Rica.

Ruíz, Reynaldo y Quintanilla, José Ramón. (2009). NNN. Enrique Flores habla de los ticos. Recuperado de http://www.youtube.com/watch?v=aNuOWaa7ftg

Sandoval García, Carlos. (2006). Fuera de juego. Fútbol, identidades nacionales y masculinidades en Costa Rica. San José: Editorial Universidad de Costa Rica.

Smith, Barbara. (1993 [1982]). Homophobia: Why Bring It Up? En Henry Abelove; Michéle Aina Barale y David Halperin (eds.) Lesbian and Gay Studies Reader (pp. 99-102). New York: Routledge.

Terminière, Pauline. (17 de mayo de 2003). Entrevista con Christine Delphy: el género, sexo social. Rebelión Pan y Rosas. Recuperado de http://www. rebelion.org/hemeroteca/mujer/030517terminiere.htm

Warner, Michael. (1994). Introduction. En Michael Warner (ed.) Fear of a Queer Planet. Queer Politics and Social Theory. Second Printing (pp. vii-xxxi). Minneapolis: University of Minnesota Press.

Wodak, Ruth. (2003). De qué trata el análisis crítico del discurso (ACD). Resumen de su historia, sus conceptos fundamentales y sus desarrollos. En Ruth Wodak y 110 Michael Meyer (comps.) Métodos de análisis crítico del discurso. Traducción de 
Tomás Fernández Aúz y Beatriz Eguibar (pp. 17-34). Barcelona: Editorial Gedisa S.A.

Zimmerman, Marc y Baeza Ventura, Gabriela. (2009). Introducción. En Marc Zimmerman y Gabriela Baeza Ventura (eds.) Estudios culturales centroamericanos en el nuevo milenio (pp. xiii-xxxv). San José: Editorial Universidad de Costa Rica. 